Electronic Supporting Information for

\title{
Novel extrusion-microdrilling approach to fabricate calcium phosphate-based bioceramic scaffolds enabling fast bone regeneration
}

Fupo He $\mathrm{He}^{\mathrm{a}, \mathrm{b}, 1, *}$, Teliang Lu ${ }^{\mathrm{c}, 1}$, Xibo Fang ${ }^{\mathrm{a}}$, Songheng Feng ${ }^{\mathrm{a}}$, Shenglei Feng ${ }^{\mathrm{d}}$,Ye Tian ${ }^{\mathrm{a}}$, Yanhui $\mathrm{Li}^{\mathrm{a}}$, Fei Zuo ${ }^{\mathrm{a}}$, Xin Deng ${ }^{\mathrm{a}, \mathrm{b}}$, Jiandong Ye $\mathrm{C}^{\mathrm{c}, *}$

a School of Electromechanical Engineering, Guangdong University of Technology, Guangzhou 510006, People's Republic of China

b Jihua Laboratory, Foshan 528200, People's Republic of China

${ }^{c}$ School of Materials Science and Engineering, South China University of Technology, Guangzhou 510641, People's Republic of China

${ }^{d}$ School of Civil Engineering, Hebei University of Engineering, Handan 056038, People's Republic of China

${ }^{1}$ Equal contribution to this paper

${ }^{*}$ Correspondence to: fphe@gdut.edu.cn (F. He), jdye@scut.edu.cn (J. Ye). 
1. Procedure of preparing bioceramic extracts

The bioceramic extracts were prepared to investigate how the ions released from the bioceramic scaffolds affect the cell response. The extracts were prepared following the protocols of International Standard Organization (ISO 10993-12). In brief, the EM-TCP and EM-TCP/SrPG scaffolds were pulverized, and immersed in the high-glucose Dulbecco's modified eagle's medium (H-DMEM; Gibco, USA) at $37{ }^{\circ} \mathrm{C}$, and the ratio of bioceramic mass to medium volume was $0.2 \mathrm{~g} \mathrm{~mL}^{-1}$. After $24 \mathrm{~h}$, the mixtures were centrifuged to collect the supernatant. For sterilization, the collected extracts were filtrated through $220 \mathrm{~nm}$ filtering membranes.

2. Procedure of evaluating in vitro degradation profiles of scaffolds

The in vitro degradation of the E-TCP, EM-TCP and EM-TCP/SrPG scaffolds was evaluated by immersion in the Tris- $\mathrm{HCl}$ solution $\left(37^{\circ} \mathrm{C}, \mathrm{pH} 7.4\right)$. The ratio of scaffold weight to solution volume was $0.05 \mathrm{~g} \mathrm{~mL}^{-1}$. The solutions were renewed every two weeks. After immersion for scheduled time, the scaffold samples were taken out, washed with deionized water 3 times, air-dried at $70{ }^{\circ} \mathrm{C}$ for $24 \mathrm{~h}$. The degradation rate (DR) was calculated using the following equation:

$$
\mathrm{DR} \%=100 \% \times\left(\mathrm{W}_{0}-\mathrm{W}_{\mathrm{t}}\right) / \mathrm{W}_{0}
$$

where $\mathrm{W}_{0}$ is the weight of the samples before immersion in solution, and $\mathrm{W}_{\mathrm{t}}$ denotes the weight of the dried samples after immersion for the scheduled time. The compressive strength of scaffolds after immersion for scheduled time was measured employing the static mechanical testing machine. 
Table S1. Primer sequences used for RT-PCR in the study.

\begin{tabular}{|c|c|}
\hline Gene & Primer sequences \\
\hline ALP & $\begin{array}{l}\text { Forward: 5'-TGCCTACTTGTGTGGCGTGAA-3' } \\
\text { Forward: 5'-TCACCCGAGTGGTAGTCACAATG-3' }\end{array}$ \\
\hline Col I & $\begin{array}{l}\text { Forward: 5'-ATGCCGCGACCTCAAGATG-3' } \\
\text { Reverse: 5'-TGAGGCACAGACGGCTGAGTA-3' }\end{array}$ \\
\hline Runx2 & $\begin{array}{l}\text { Forward: 5'-CACTGGCGGTGCAACAAGA-3' } \\
\text { Forward: 5'-TTTCATAACAGCGGAGGCATTTC-3' }\end{array}$ \\
\hline BSP & $\begin{array}{l}\text { Forward: 5'-AGAACAATCCGTGCCACTCACTC-3' } \\
\text { Forward: 5'-AGTAGCGTGGCCGGTACTTAAAGA-3' }\end{array}$ \\
\hline OPN & $\begin{array}{l}\text { Forward: 5'-TGCAAACACCGTTGTAACCAAAAGC-3' } \\
\text { Forward: 5'-TGCAGTGGCCGTTTGCATTTCT-3' }\end{array}$ \\
\hline OCN & $\begin{array}{l}\text { Forward: 5'-AGCAGCTTGGCCCAGACCTA-3' } \\
\text { Reverse: 5'-TAGCGCCGGAGTCTGTTCACTAC-3' }\end{array}$ \\
\hline NFATc 1 & $\begin{array}{l}\text { Forward: 5'-GGTAACTCTGTCTTTCTAACCTTAAGCTC-3' } \\
\text { Reverse:5'-GTGATGACCCCAGCATGCACCAGTCACAG-3' }\end{array}$ \\
\hline Car2 & $\begin{array}{l}\text { Forward: 5'-CAAGTCTCTTCGAGTACATTGCC-3' } \\
\text { Reverse: 5'-CCTGGTTCTGTATGTGCAGGTA-3' }\end{array}$ \\
\hline c-Fos & $\begin{array}{l}\text { Forward: 5'-CCAAGCGGAGACAGATCAACTT-3' } \\
\text { Reverse: 5'-TCCAGTTTTTCCTTCTCTTTCAGCAGAT-3' }\end{array}$ \\
\hline TRAP & $\begin{array}{l}\text { Forward: 5'-TACCTGTGTGGACATGACC-3' } \\
\text { Reverse: 5'-CAGATCCATAGTGAAACCGC-3' }\end{array}$ \\
\hline MMP9 & $\begin{array}{l}\text { Forward: 5'-TCCAGTACCAAGACAAAGCCTA-3' } \\
\text { Reverse: 5'-TTGCACTGCACGGTTGAA-3' }\end{array}$ \\
\hline GAPDH & $\begin{array}{l}\text { Forward: 5'-TGTGTCCGTCGTGGATCTGA-3' } \\
\text { Reverse: 5'-TTGCTGTTGAAGTCGCAGGAG-3' }\end{array}$ \\
\hline
\end{tabular}




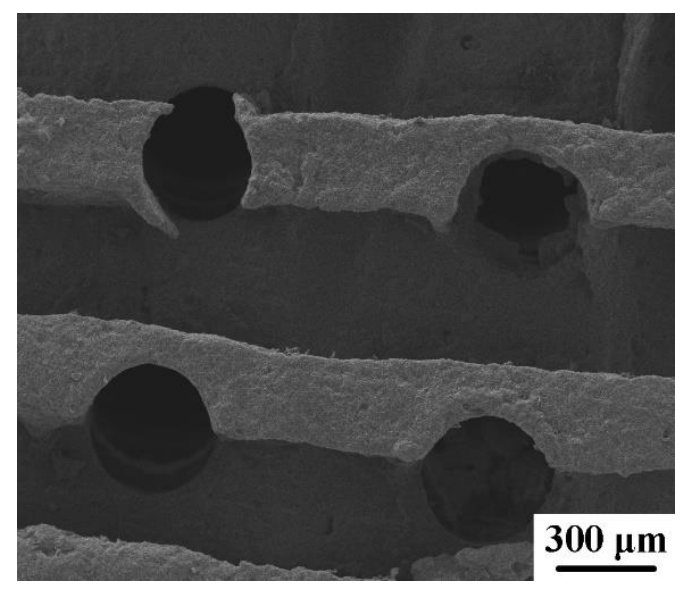

Figure S1. SEM images of the EM-TCP scaffolds in which the microdrilled pores were situated at the cross section of extruded pore walls.
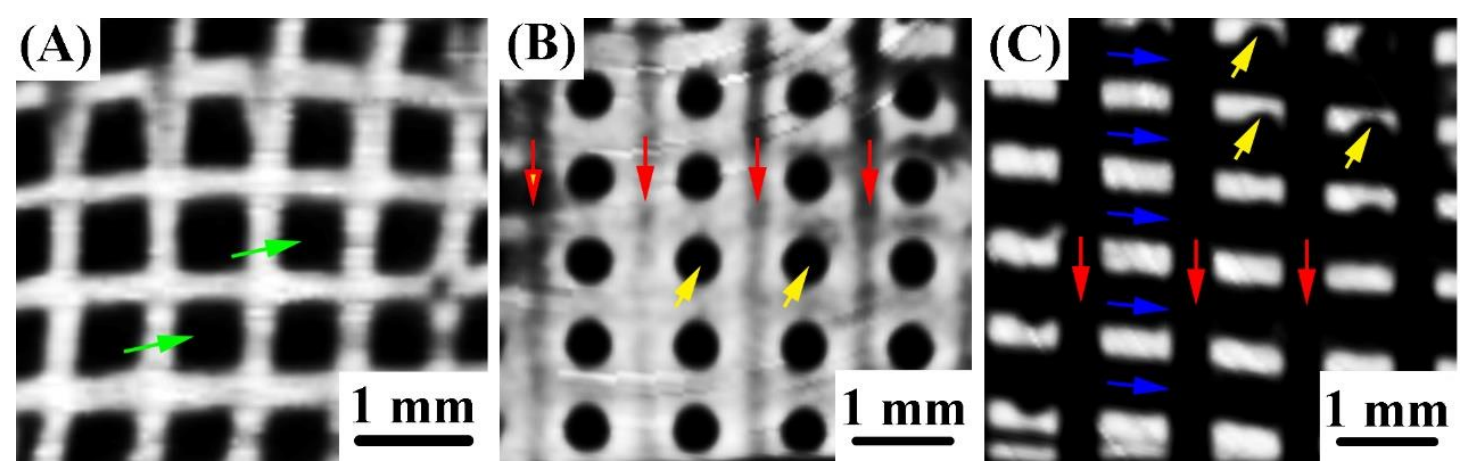

Figure. S2. Cross-sectional $\mu$-CT images of the EM-TCP/SrPG. (A) Cross-sectional image showing the extruded square macropores and their configuration; (B) cross-sectional image showing the microdrilled round macropores and their configuration; (C) cross-sectional image showing the extruded macropores and microdrilled macropores, and their configuration. Green arrow points to the square macropores formed by extrusion; yellow arrow points to the round macropores formed by microdrilling; red arrow points to the direction of round macropores; blue arrow points to the direction of square macropores. 


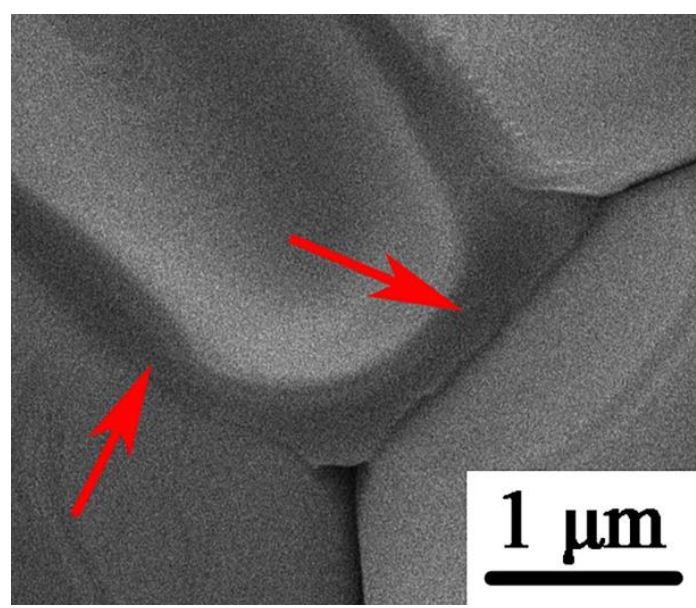

Figure S3. SEM image of the EM-TCP/SrPG scaffolds. Red arrow points to the glassy phase at the grain boundary of EM-TCP/SrPG.

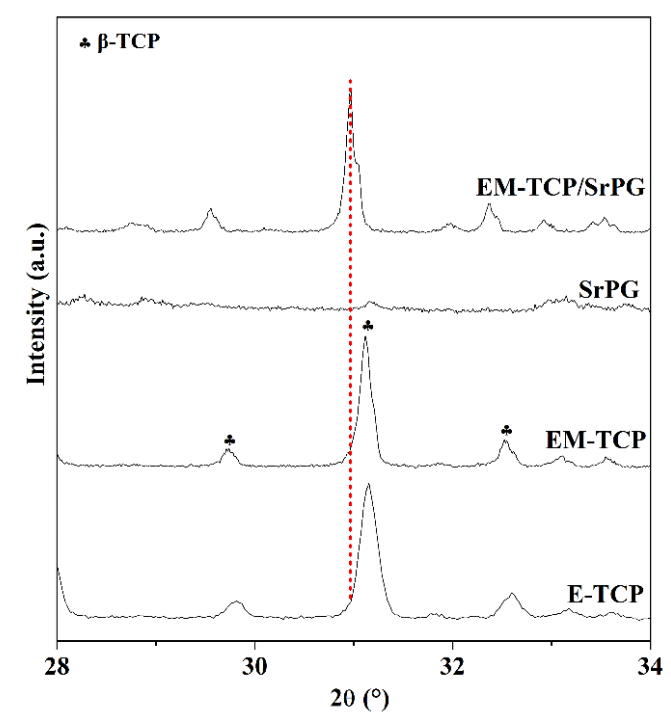

Figure S4. A section of XRD patterns of the E-TCP, EM-TCP, SrPG and $\mathrm{EM}-\mathrm{TCP} / \mathrm{SrPG}$ in the range of $28^{\circ}$ to $34^{\circ}$ for $2 \theta$. The peak positions of $\beta$-TCP in the pattern of EM-TCP/SrPG shifted to the lower angles, as compared with those of E-TCP and EM-TCP. 


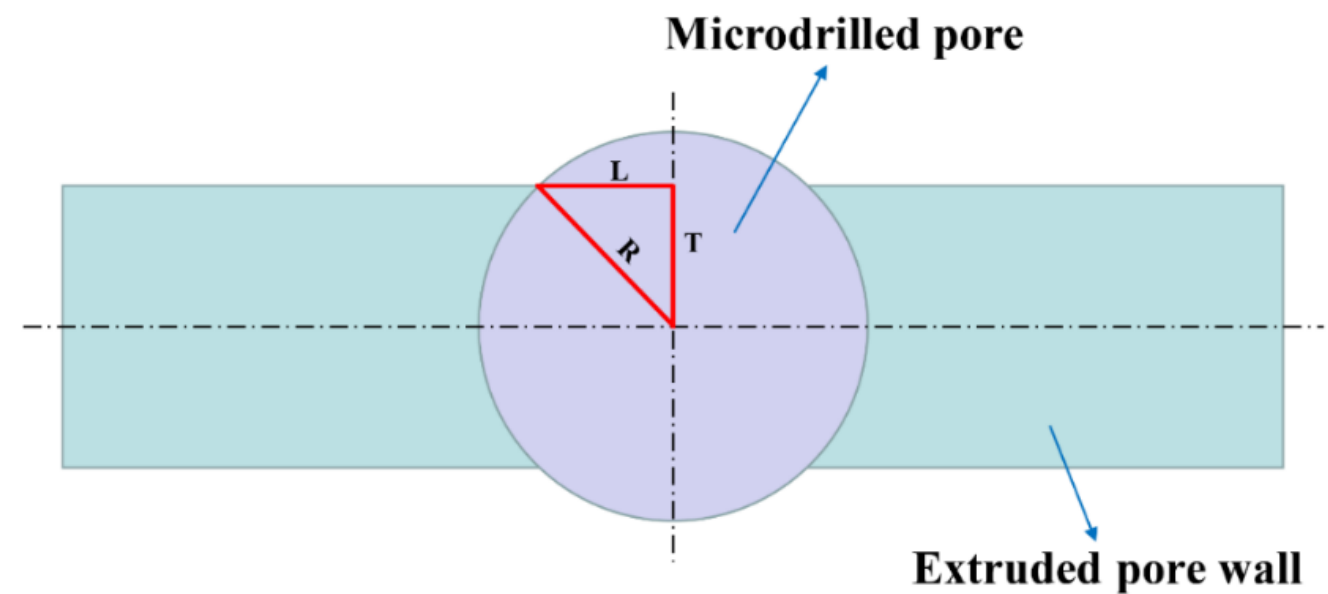

Figure S5. Schematic of positional relation between the microdrilled pores and cross section of extruded pore wall, when the center of microdrilled pores were situated at the center line of cross section of the pore wall. The diameter of microdrilled pores (2R) of green body was $600 \mu \mathrm{m}$; the thickness of pore wall (2T) was $480 \mu \mathrm{m}$; the size of interconnected pore (2L) between microdrilled pore and extruded pore wall was $360 \mu \mathrm{m}$, as calculated by the equation as follow: $\mathrm{L}=\sqrt[2]{\mathrm{R}^{2}-\mathrm{T}^{2}}$. After sintering, the pore size was decreased by less than $15 \%$ for all the ceramic scaffolds. That is to say, the size of interconnected pore size of between the microdrilled pores and extruded pores for all the bioceramic scaffolds was larger than $300 \mu \mathrm{m}$. The interconnected pore size would become larger when the center of microdrilled pores were not situated at the center line of cross section of the pore wall. 

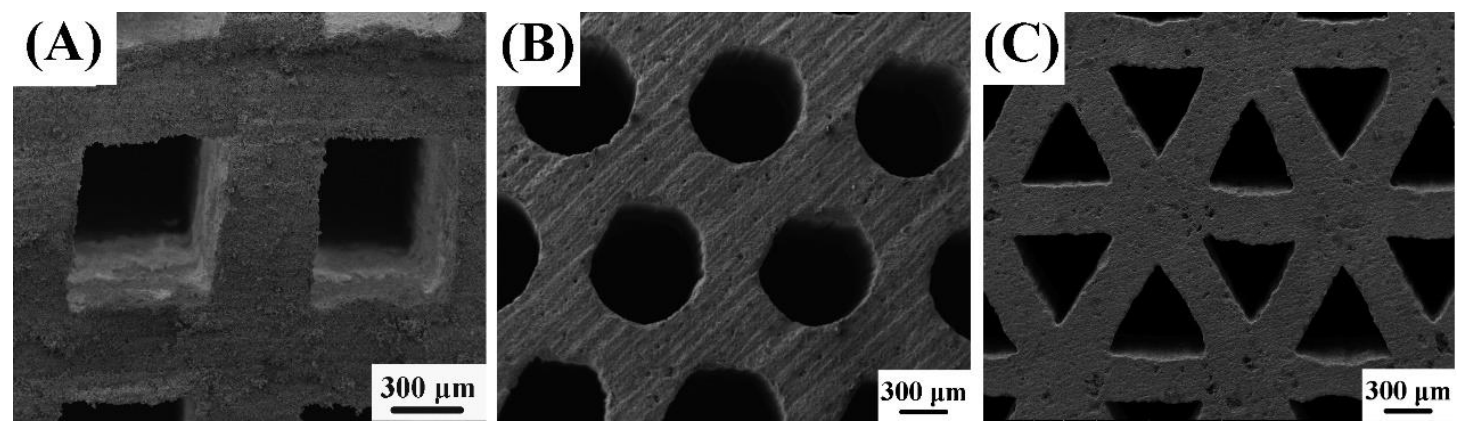

Figure S6. SEM images of the extruded pores with various size and pore shape (A: square; B: round; C: triangle).
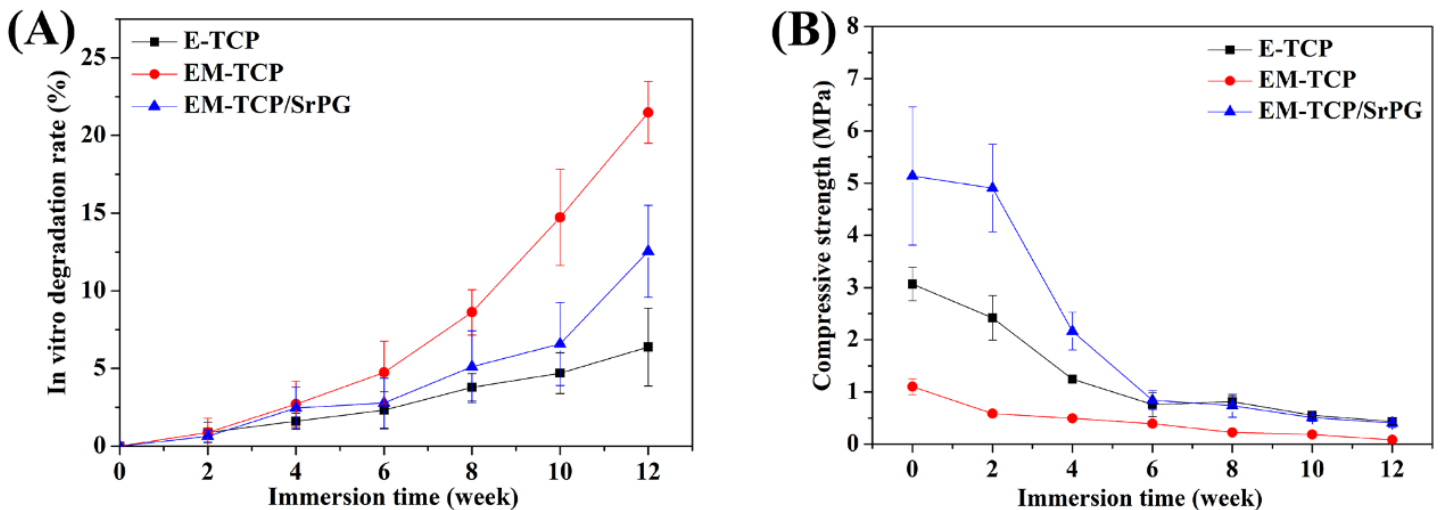

Figure S7. In vitro degradation rate (A) and corresponding compressive strength variation (B) of the E-TCP, EM-TCP and EM-TCP/SrPG scaffolds. 